\title{
Steady-shear and viscoelastic properties of cellulose nanofibril-nanoclay dispersions
}

\author{
Yingxin Liu $\cdot$ Korneliya Gordeyeva $\cdot$ Lennart Bergström
}

Received: 20 September 2016/ Accepted: 27 January 2017/Published online: 8 February 2017

(C) The Author(s) 2017. This article is published with open access at Springerlink.com

\begin{abstract}
We have investigated the steady-shear and viscoelastic properties of composite dispersions of cellulose nanofibrils (CNFs) with medium or high charge density and two different nanoclays, viz. rodlike sepiolite or plate-like bentonite. Aqueous dispersions of CNFs with medium charge density displayed significantly lower steady-state viscosity and storage modulus but higher gelation threshold compared with CNFs with high charge density. Dynamic light scattering (DLS) results showed that the apparent hydrodynamic radius of bentonite particles increased when CNFs were added, implying that CNFs adsorbed onto the amphoteric edges of the plate-like bentonite particles. The sepiolite network in CNF-sepiolite dispersions was relatively unaffected by addition of small amounts of CNFs, and DLS showed that the hydrodynamic radius of sepiolite did not change when CNFs were added. Addition of CNFs at concentrations above the gelation threshold resulted in drastic
\end{abstract}

Electronic supplementary material The online version of this article (doi:10.1007/s10570-017-1211-3) contains supplementary material, which is available to authorized users.

Y. Liu · K. Gordeyeva · L. Bergström ( $ه)$

Department of Materials and Environmental Chemistry, Stockholm University, 10691 Stockholm, Sweden

e-mail: lennart.bergstrom@mmk.su.se

Y. Liu

Wallenberg Wood Science Center, KTH,

10044 Stockholm, Sweden decrease of the steady-shear viscosity of the sepiolite dispersion, suggesting that the sepiolite network disintegrates and the rod-like clay particles are aligned also at low shear rate. The relative change in the rheological properties of the clay-based dispersions was always greater on addition of CNFs with high compared with medium charge density. This study provides insight into how the rheology of CNFnanoclay dispersions depends on both the nanoclay morphology and the interactions between the nanoclay and nanocellulose particles, being of relevance to processing of nanocellulose-clay composites.

Keywords Cellulose nanofibrils $\cdot$ Nanocellulose dispersions $\cdot$ Bentonite $\cdot$ Sepiolite $\cdot$ Viscoelasticity

\section{Introduction}

Cellulose nanofibrils (CNFs), extracted from wood, marine animals, and bacteria, are a sustainable nanomaterial that combines high specific stiffness, high surface area, low density, optical transparency, and flexible surface chemistry (Klemm et al. 2011; Moon et al. 2011; Usov et al. 2015). Among a variety of mechanical and chemical approaches (Moon et al. 2011; Pääkkö et al. 2007), 2,2,6,6-tetramethyl-1piperidinyl-1-oxyl (TEMPO)-mediated oxidation is a recently developed and already widely used route to prepare colloidally stable CNFs (Isogai et al. 2011; Saito et al. 2006). CNFs and other forms of 
nanocellulose are of interest for use in a wide range of applications, e.g., as additives in food, cosmetics, and paints, and as rheology modifiers (Klemm et al. 2011). CNFs can also be used to produce strong and transparent nanopapers (Henriksson et al. 2008), flexible filaments (Hakansson et al. 2014; Iwamoto et al. 2011), lightweight foams (Gordeyeva et al. 2016; Wicklein et al. 2016), and hydrogels (Klemm et al. 2011; Munier et al. 2016).

It is clear that successful production of CNF-based composite materials relies on the ability to process and assemble CNF-based aqueous dispersions (Abdul Khalil et al. 2012; Siró and Plackett 2010). Previous work has shown that aqueous dispersions of slender and kinked CNFs display non-Newtonian rheological behavior characterized by shear thinning and pronounced viscoelastic behavior at concentrations above the gelation threshold (de Kort et al. 2016; Jowkarderis and van de Ven 2015; Martoia et al. 2016). Recent studies have investigated the effects of pulp source (Tanaka et al. 2016), fibril dimension and concentration (Agoda-Tandjawa et al. 2010), mechanical treatments (Pääkkö et al. 2007), ionic strength (Dong et al. 2013), pH (Fall et al. 2013), as well as addition of amphiphilic molecules (Quennouz et al. 2016) on the rheology of CNF dispersions. Additionally, CNFs can also assist in preparation of homogeneous dispersions of a variety of fillers and pigments, such as carbon nanotubes (Hamedi et al. 2014), reduced graphene oxide (Duan et al. 2016), $\mathrm{TiO}_{2}$ (Schütz et al. 2012), and $\mathrm{CaCO}_{3}$ (Lourenço et al. 2016) nanoparticles.

Nanoclays (Bailey et al. 2015) are commonly used as an additive in CNF-based composite materials to improve, e.g., their mechanical properties (Gabr et al. 2013; Wang et al. 2014) or fire retardancy (Liu et al. 2011; Wicklein et al. 2015), and to tailor the selectivity and flux of membranes (Zheng et al. 2014). Notably, it has been shown that CNF-bentonite dispersions display properties suitable for use as drilling fluids (Li et al. 2015). However, comprehensive studies on the steady-shear and viscoelastic properties of such CNF-nanoclay dispersions are rare, and previous rheological studies have mainly focused on dispersions of plate-like nanoclays and moderately charged or uncharged nanocellulose particles (Abend and Lagaly 2000; Li et al. 2015; Ming et al. 2016; Nechyporchuk et al. 2014).

In this study, we evaluated the steady-shear and viscoelastic properties of aqueous dispersions of plate- like bentonite or rod-like sepiolite clay nanoparticles together with CNFs with moderate or high charge density. The effect of the CNF content on the viscosity under shear and the storage modulus at quiescent conditions of the nanoclay dispersions is found to be related to the interactions between the CNFs and the different types of clay particle.

\section{Experimental procedures}

\section{Materials}

Never-dried sulfite softwood pulp (Domsjö dissolving pulp, 60/40 spruce/pine, Sweden) was the cellulose source. Sodium hypochlorite (Alfa Aesar), 2,2,6,6tetramethyl-1-piperidinyloxy free radical (TEMPO, $\geq 98 \%$, Alfa Aesar), sodium hydroxide ( $\geq 99.2 \%$, VWR Chemicals), sodium bromide (BioUltra, $\geq 99.5 \%$, Sigma Aldrich), (3-aminopropyl)triethoxysilane (Sigma Aldrich, $99 \%$ ), sodium bentonite, and sepiolite (Sigma Aldrich) were used as received.

\section{Preparation of CNF dispersions}

Cellulose nanofibrils were prepared using the method developed by Saito et al. (2006). Oxidized pulp was washed with deionized water (DI) water until the conductivity of the filtrate was below $5 \mu \mathrm{S}$. After diluting to $1.0 \mathrm{wt} \%$, the pulp dispersion was disintegrated using a high-pressure (1600 bar) microfluidizer (M-110EH, Microfluidics) with 200- and 100- $\mu \mathrm{m}-$ wide chambers connected in series for eight times. The charge density (carboxyl groups per gram of pulp) was characterized by conductometric titration (Fall et al. 2011) with relative error below $5 \%$. CNFs with medium (649 $\pm 34 \mu \mathrm{mol} / \mathrm{g}, \mathrm{MCNFs})$ and high charge density $(1030 \pm 38 \mu \mathrm{mol} / \mathrm{g}$, HCNFs) were prepared.

Aqueous dispersions with CNF concentration of $0.1,0.25,0.5,0.75$, and $1.0 \mathrm{wt} \%$ were prepared by dilution of stock dispersion, agitated using an ULTRA-TURRAX (IKA), and degassed by vacuum before each measurement.

Preparation of CNF-nanoclay dispersions

Sodium bentonite and sepiolite were purified by dialysis in flowing DI water for 3 days until the $\mathrm{pH}$ stabilized at around 6.8. Aqueous clay dispersions 
with concentration of $4.0 \mathrm{wt} \%$ were prepared by mixing purified clay with DI water, followed by 10 days of continuous agitation at $500 \mathrm{rpm}$ using an IKA magnetic stirrer. Composite dispersions were prepared by mixing CNF and nanoclay dispersions to obtain dispersions with nanoclay concentration of $2.0 \mathrm{wt} \%$ (with respect to the aqueous phase) and CNF concentration of $0.1,0.25$, and $0.5 \mathrm{wt} \%$ (also with respect to the aqueous phase). The solid contents of the CNF-nanoclay dispersions are summarized in Table S1. The composite dispersions (at $\mathrm{pH} 6.8$ ) were agitated using an ULTRA-TURRAX (IKA T25) and degassed by vacuum before measurements.

\section{Characterization}

The diameter and length distribution of the CNFs were characterized by atomic force microscopy (AFM, tapping mode, Veeco Multimode V, USA). CNFs were deposited on mica substrates coated with (3-aminopropyl)triethoxysilane. The morphology of sepiolite and bentonite was also characterized by AFM. The dimensions (height and length) of the CNFs were determined by analysis of AFM images of approximate 300 fibrils using NanoScope Analysis and ImageJ software. The average and distribution of fibril length were calculated using a previously reported method (Tanaka et al. 2014).

The optical transparency of the CNF aqueous dispersions was characterized using an ultraviolet/ visible/near infrared (UV/VIS/NIR) spectrophotometer (Lambda 19, PerkinElmer) in quartz cuvettes.

Rheological measurements were performed at $25^{\circ} \mathrm{C}$ using a Physica MCR 301 rheometer (Anton Paar) equipped with a smooth cone-on-plate geometry (CP25-2-SN7617, diameter $25 \mathrm{~mm}, 2^{\circ}$ nominal angle, gap height $0.5 \mathrm{~mm}$ ). To minimize evaporation during measurements, we used an evaporation blocker and a thin layer of low-density mineral oil spread on the periphery of the samples. Steady-shear measurements were performed from 0.1 to $1000 \mathrm{~s}^{-1}$. Oscillatory frequency sweeps were performed at angular frequency from 0.1 to $60 \mathrm{rad} \mathrm{s}^{-1}$ with $5 \%$ strain, which is within the linear viscoelastic regime.

Digital photographs of tilted dispersions in glass vials (diameter $12 \mathrm{~mm}$, height $32 \mathrm{~mm}$ ) were taken after $180 \mathrm{~s}$. The dispersions were allowed to equilibrate in nontilted position overnight prior to the measurements.

Dynamic light scattering measurements were performed using a Zetasizer Nano ZS (Malvern Instruments Ltd., UK), equipped with a 530-nm laser and backscatter detector (at angle of $173^{\circ}$ ). Normalized

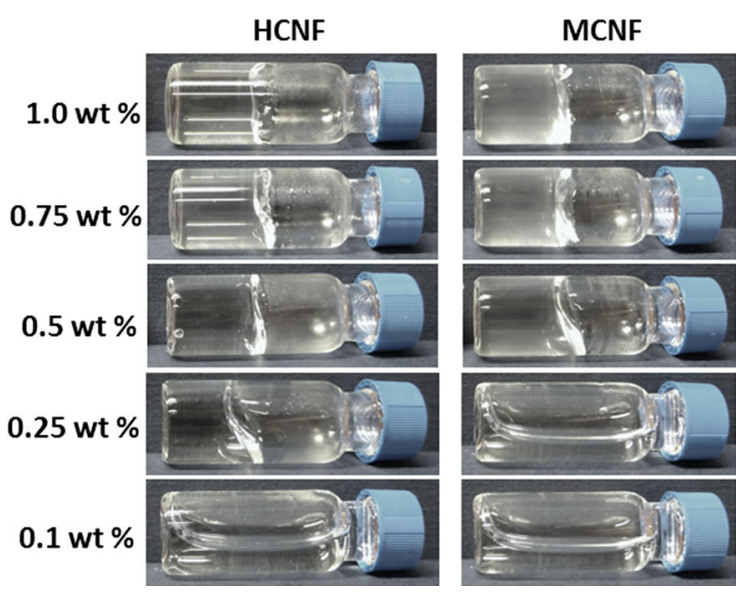

Fig. 2 Digital photographs of $90^{\circ}$ tilted vials of $\mathrm{CNF}$ aqueous dispersions (MCNFs and HCNFs with charge density of $649 \pm 34$ and $1030 \pm 38 \mu \mathrm{mol} / \mathrm{g}$, respectively) at concentration of $0.1,0.25,0.5,0.75$, and $1.0 \mathrm{wt} \%$
Fig. 1 AFM images of a MCNFs and $\mathbf{b}$ HCNFs
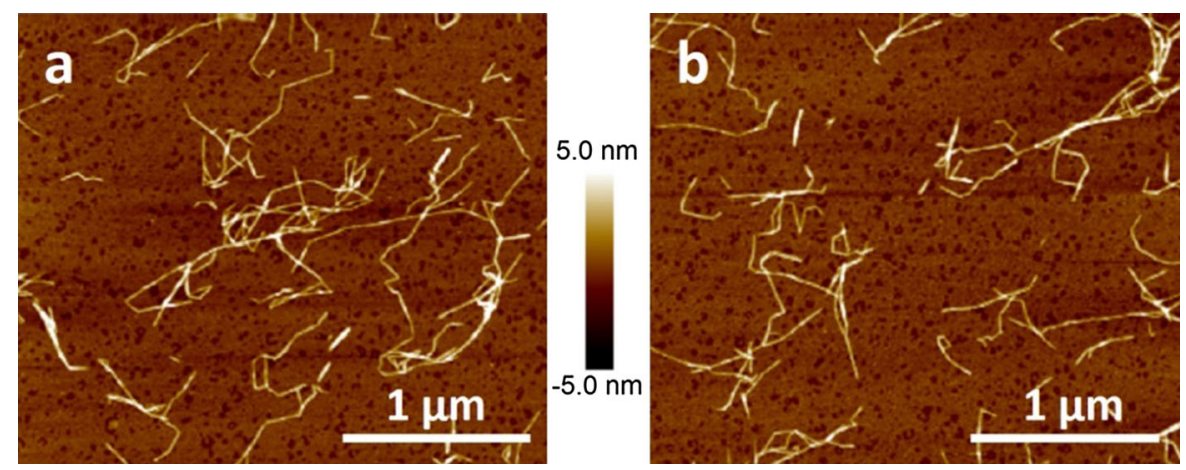
intensity correlation curves and particle size data were averaged from 10 to 20 measurements. The dynamic light scattering measurements were performed on $\mathrm{CNF}$-nanoclay and nanoclay dispersions that had been diluted 20 times from the dispersions used in rheology tests.

The zeta potential of nanoclays in aqueous dispersion at different $\mathrm{pH}$ was determined from an average of 10 measurements of 30 runs each, also with a Zetasizer Nano ZS (Malvern Instruments Ltd., UK).

\section{Results and discussion}

We prepared CNFs with medium and high charge density by TEMPO-mediated oxidation and high-
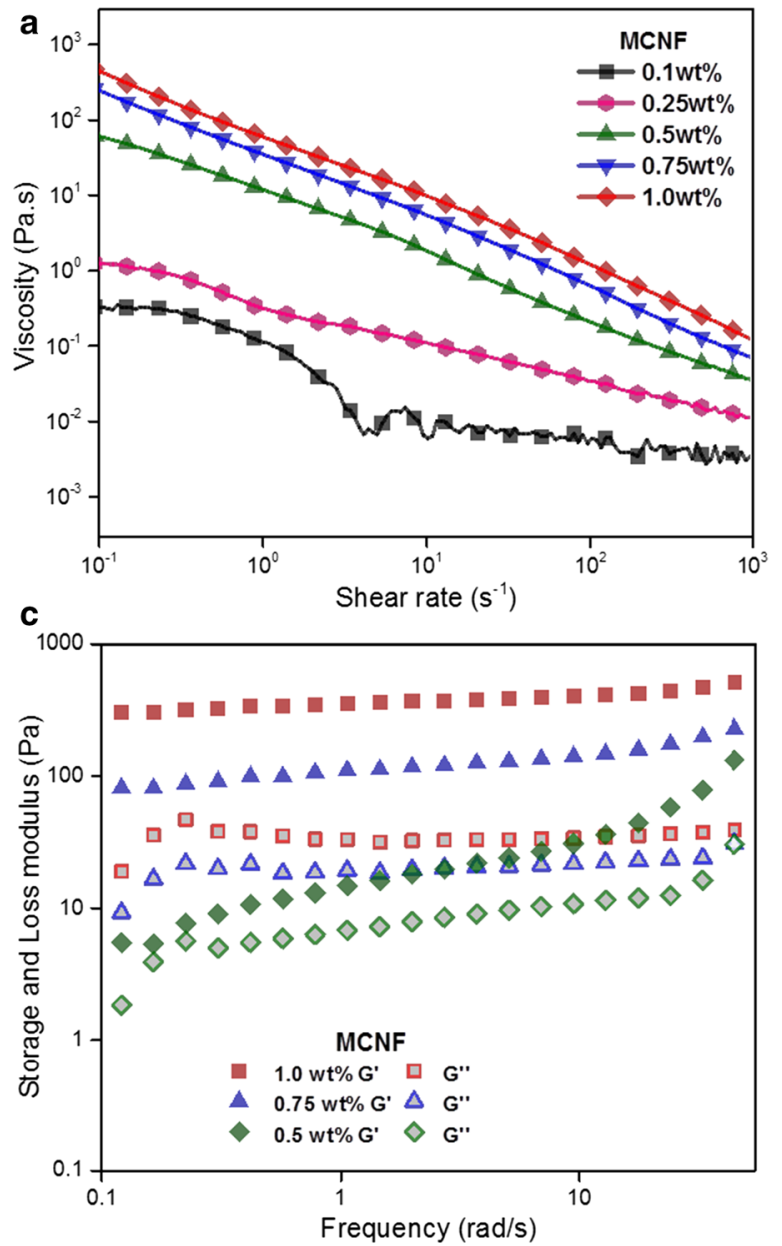

Fig. 3 Steady-shear and viscoelastic measurements of CNF dispersions with concentration of $0.1,0.25,0.5,0.75$, and 1.0 wt\%: viscosity as function of shear rate for a MCNF and pressure homogenization, and characterized their dimensions by atomic force microscopy (AFM) (Fig. 1). The CNFs displayed well-defined fibril diameter of $2.35 \pm 0.77$ and $2.30 \pm 0.65 \mathrm{~nm}$ for MCNFs and HCNFs, respectively, in correspondence with previous studies (Gordeyeva et al. 2016). The average and distribution of the length of both types of fibril are summarized in Fig. S1 and Table S2.

Figure 2 shows that dispersions of $\mathrm{HCNF}$ at concentrations above $0.25 \mathrm{wt} \%$ could form selfstanding hydrogels that did not flow when tilted by $90^{\circ}$, while the critical gelation concentration was $0.5 \mathrm{wt} \%$ for the MCNFs with lower charge density. Hence, the gelation threshold (where the material changes from a solution to a hydrogel) of the aqueous CNF dispersions depends on both the charge density of
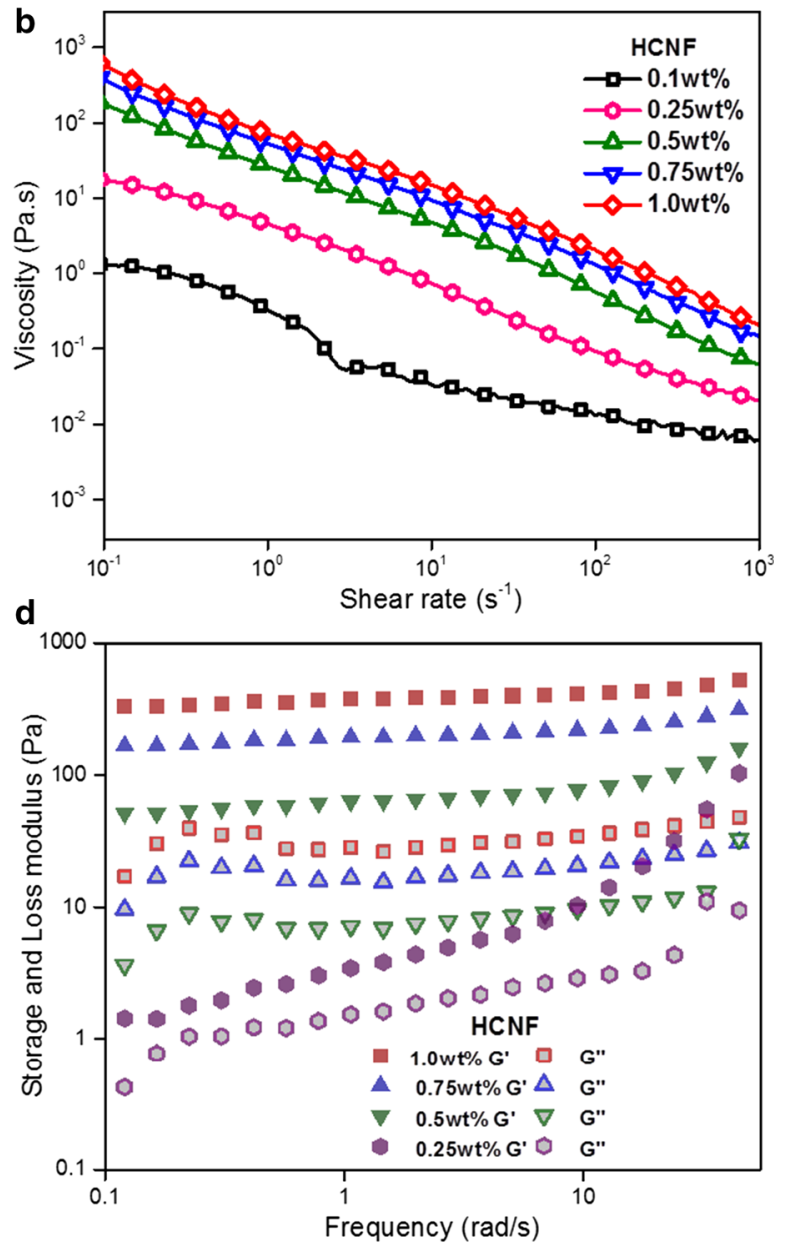

b HCNF dispersions, and frequency sweep (at $5 \%$ strain) for c MCNF and d HCNF dispersions (storage modulus $G^{\prime}$, loss modulus $G^{\prime \prime}$ ) 
the fibrils and their concentration. UV/VIS measurements (Fig. S2) showed that the HCNF dispersions had higher optical transparency than the MCNF dispersions at the same concentration, suggesting that the formed contained fewer light-scattering aggregates and were more defibrillated. Strong repulsion between the electric double layers of the HCNFs thus promotes homogeneous distribution of the nanofibrils with low gelation threshold.

Figure 3 displays the typical shear-thinning behavior of CNF dispersions. The dispersions showed a constant level of the degree of shear thinning, characterized by the slope of the log-log shear curves, over a shear rate range spanning at least two decades, when the CNF concentration was equal to or above the gelation threshold, i.e., $0.5 \mathrm{wt} \%$ for MCNFs or $0.25 \mathrm{wt} \%$ for HCNFs, whereas the shear thinning seemed to be discontinuous at low concentration $(0.1 \mathrm{wt} \%)$. We found that the HCNF dispersions always displayed substantially higher viscosity than the MCNF dispersions; the viscosity at $10 \mathrm{~s}^{-1}$ was about 2.5 times higher for HCNF compared with MCNF dispersion at concentration of $0.5 \mathrm{wt} \%$. This effect is probably related to the higher effective volume of the CNFs with high compared with medium charge density (Blakeney 1966; Johansson et al. 2011; Mazeau 2011). The more pronounced shear-thinning behavior of MCNF compared with HCNF dispersions
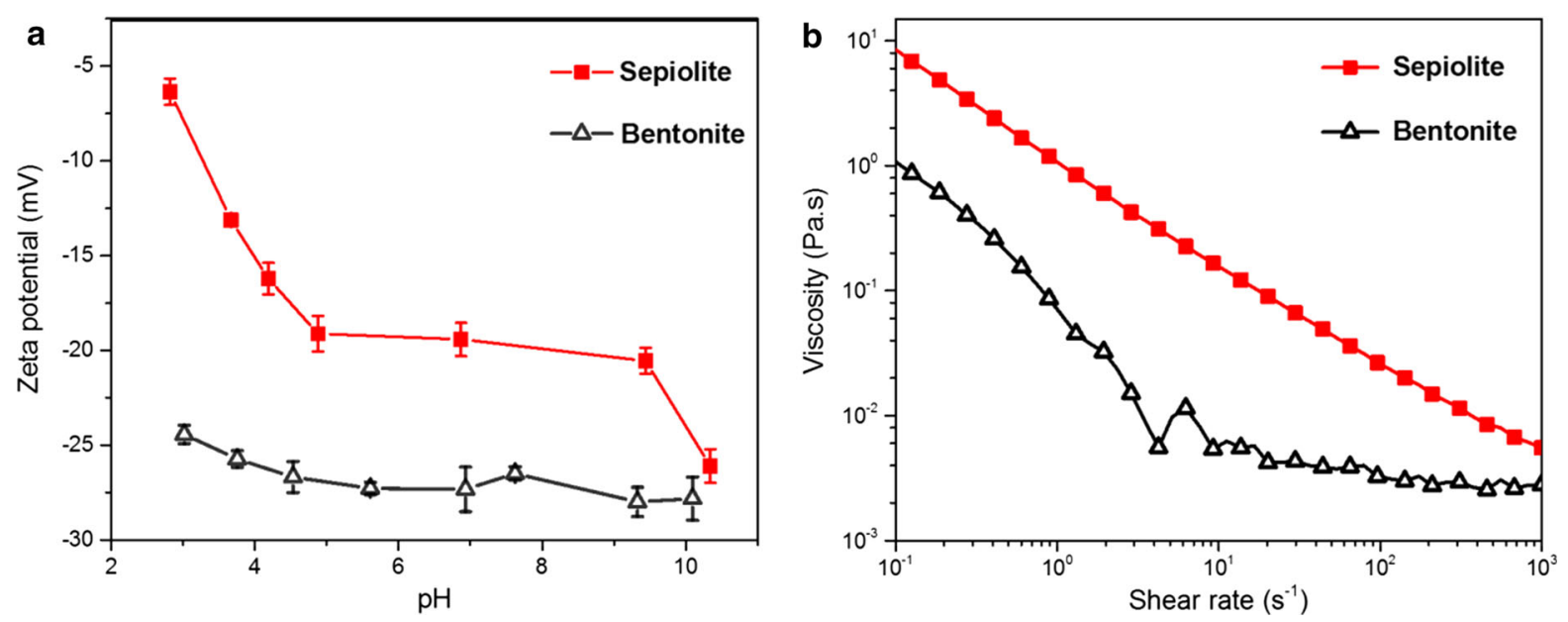

Fig. 4 Characterization of bentonite and sepiolite: a zeta potential and $\mathbf{b}$ steady-shear curves of aqueous clay dispersions (2.0 wt $\%)$ at $\mathrm{pH} 6.8$

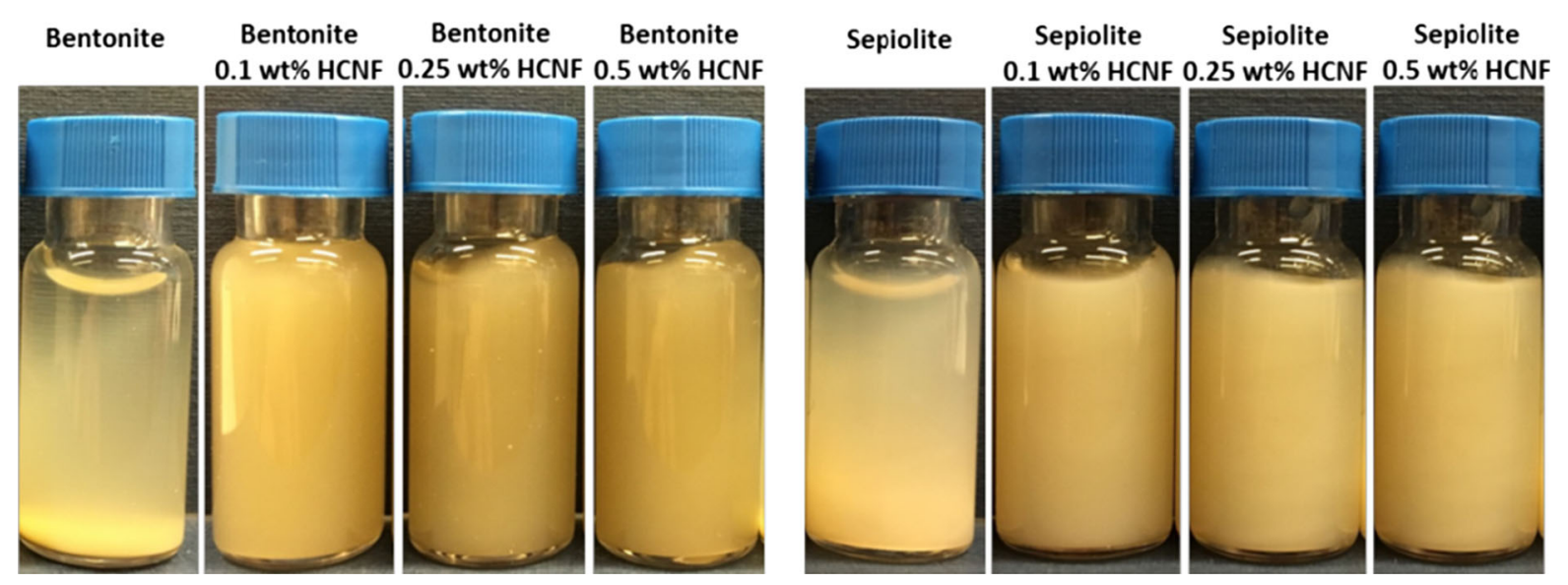

Fig. 5 Digital photographs of HCNF-nanoclay dispersions 10 days after preparation (2.0 wt $\%$ nanoclay with $0.1,0.25$, or 0.5 wt $\%$ HCNFs) 
could originate from shear-induced breakup of flocculated fibrils. Indeed, the lower optical transmittance of MCNF compared with HCNF dispersions in Fig. S2 corroborates that a fraction of the MCNFs may be slightly flocculated.

The shear stress was always larger than the estimated yield stress (Dimic-Misic et al. 2013) above shear rate of $0.02 \mathrm{~s}^{-1}$ (Fig. S3), which suggests that wall slip was insignificant. Oscillatory viscoelastic measurements showed that both HCNF and MCNF dispersions were viscoelastic above the gelation threshold (Nechyporchuk et al. 2015; Quennouz et al. 2016).

We characterized the $\mathrm{pH}$-dependent electrokinetic properties of bentonite and sepiolite, as shown in
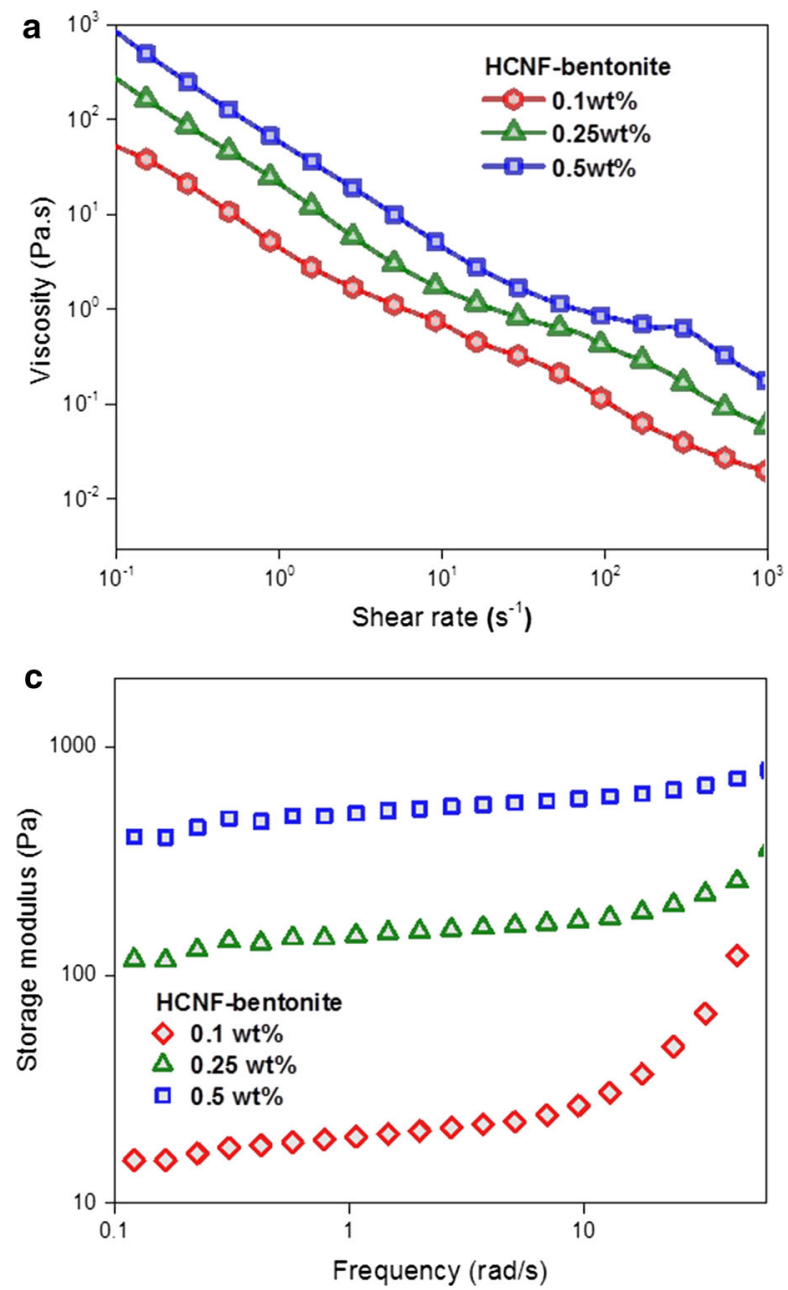

Fig. 6 Steady-shear and viscoelastic properties of HCNF-clay dispersions with different HCNF concentrations $(0.1,0.25$, and $0.5 \mathrm{wt} \%)$ : viscosity as function of shear rate for composite dispersions of HCNFs and a bentonite, $\mathbf{b}$ sepiolite; frequency
Fig. 4a. Bentonite was more negatively charged than sepiolite, with zeta potential at $\mathrm{pH} 6.8$ of -28 and $-18 \mathrm{mV}$, respectively. The negative surface charge of both bentonite and sepiolite originates primarily from isomorphic substitutions of $\mathrm{Si}^{4+}$. Such substitution in bentonite is commonly by $\mathrm{Al}^{3+}$ and $\mathrm{Fe}^{3+}$, while substitution and slow leaching of $\mathrm{Mg}^{2+}$ is responsible for the negative charge of sepiolite (Cinar et al. 2009; Luckham and Rossi 1999; Sabah et al. 2007). Bentonite is a plate-like clay with negatively charged faces and amphoteric edges dominated by $\mathrm{Al}-\mathrm{OH}$ groups (Bailey et al. 2015; Ruiz-Hitzky et al. 2013). Figure $\mathrm{S} 4$ displays the morphology and particle size of both bentonite and sepiolite. Figure $4 \mathrm{~b}$ shows that the
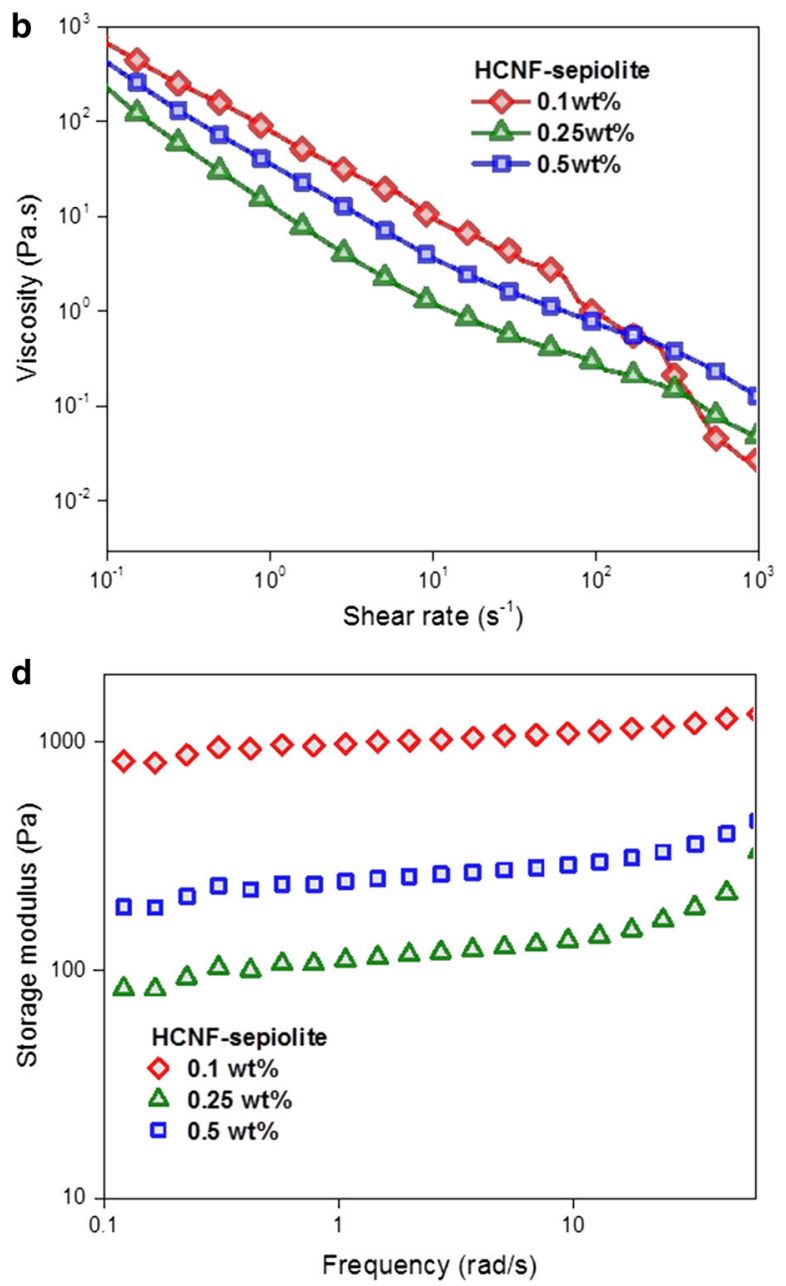

sweep ( $5 \%$ strain) of HCNFs and $\mathbf{c}$ bentonite and $\mathbf{d}$ sepiolite composite dispersions, all at constant nanoclay concentration of $2.0 \mathrm{wt} \%$ 
viscosity of aqueous dispersions of plate-like bentonite was lower compared with dispersions of rodlike sepiolite. The difference in the rheological properties is related to both the morphology and colloidal stability. The stronger shear-thinning properties of sepiolite compared with bentonite dispersions suggests that the more weakly charged rod-like sepiolite particles form a stronger three-dimensional (3D) network compared with the more highly charged, plate-like bentonite particles.

Figure 5 shows that addition of HCNFs could substantially delay the sedimentation of both bentonite and sepiolite, thus resulting in composite dispersions with long-term stability. As shown in Fig. 6, both

Table 1 Relative viscosity of $2.0 \mathrm{wt} \%$ nanoclay dispersions and viscosity ratio of $\mathrm{HCNF}$-nanoclay dispersions and nanoclay dispersions

\begin{tabular}{lllll}
\hline Shear rate $\left(\mathrm{s}^{-1}\right)$ & 0.1 & 1 & 10 & 500 \\
\hline $\begin{array}{l}\text { Relative viscosity } \\
\text { Bentonite }\end{array}$ & 1200 & 68 & 11 & 3.4 \\
$\quad$ Sepiolite & 9200 & 1100 & 190 & 7.9 \\
Viscosity ratio & & & & \\
Bentonite-HCNF(0.1) & 38 & 13 & 19 & 3.6 \\
Bentonite-HCNF(0.25) & 15 & 4.4 & 2.1 & 3.2 \\
Bentonite-HCNF(0.5) & 4.5 & 2.1 & 1.0 & 3.2 \\
Sepiolite-HCNF(0.1) & 480 & 240 & 270 & 9.0 \\
Sepiolite-HCNF(0.25) & 12 & 2.7 & 1.6 & 2.9 \\
Sepiolite-HCNF(0.5) & 2.2 & 1.3 & 1.0 & 2.2 \\
\hline
\end{tabular}

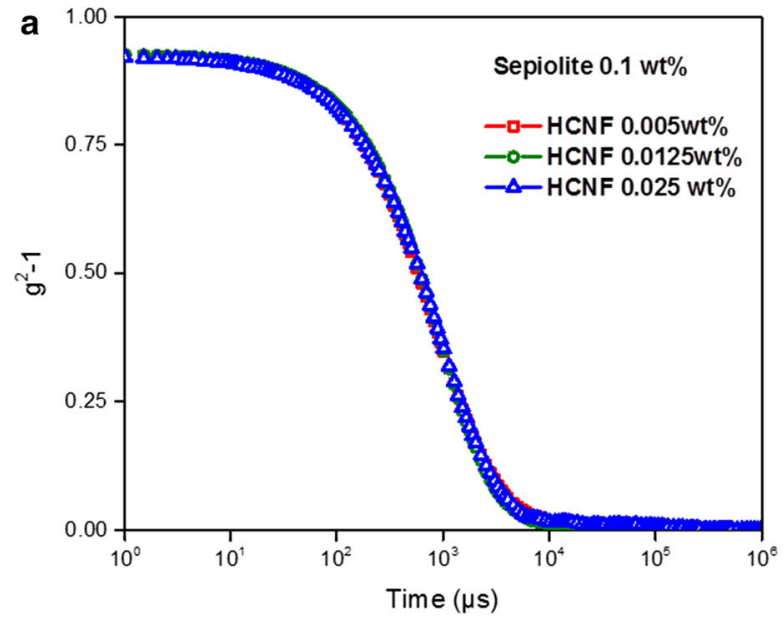

HCNF-bentonite and HCNF-sepiolite dispersions displayed pseudoplastic behavior over the investigated shear rate range. Figure $6 \mathrm{a}, \mathrm{c}$ shows that the viscosity and $G^{\prime}$ value of the HCNF-bentonite dispersions increased with the HCNF concentration. In contrast, Fig. $6 \mathrm{~b}$, d shows that the viscosity of the HCNFsepiolite dispersions was substantially higher at HCNF content of only $0.1 \mathrm{wt} \%$ (8.6 Pa.s) compared with $0.25 \mathrm{wt} \%$ (1.3 Pa.s) or $0.5 \mathrm{wt} \%$ (4.1 Pa.s) at $10 \mathrm{~s}^{-1}$, and $G^{\prime}$ decreased from 1105 to $133 \mathrm{~Pa}$ when the HCNF content was increased from 0.1 to $0.25 \mathrm{wt} \%$. The HCNF-based composite dispersions always displayed higher viscosity and $G^{\prime}$ value than the MCNFbased composite dispersions (Figs. S5, S6), similar to the CNF dispersions.

The relative viscosity (normalized to the viscosity of the continuous medium, i.e., water) of nanoclay dispersions and the corresponding viscosity ratio of the HCNF-nanoclay dispersions (normalized to the viscosity of the HCNF dispersions) are presented in Table 1. The relative viscosity of the nanoclay dispersions at high shear was 3.4 and 7.9 for the $2 \mathrm{wt} \%$ bentonite and sepiolite dispersions, respectively. The viscosity ratio of the $\mathrm{HCNF}$-bentonite dispersions (3.2-3.6) was very similar to the relative viscosity (3.4) of the bentonite dispersions at high shear rate. The relative viscosity at high shear rate was significantly higher for the rod-like sepiolite (7.9). The viscosity ratio of the $\mathrm{HCNF}$-bentonite dispersions at HCNF concentration of $0.25 \mathrm{wt} \%$ and above was between 1 and 4 at shear rate of $1 \mathrm{~s}^{-1}$ or above.

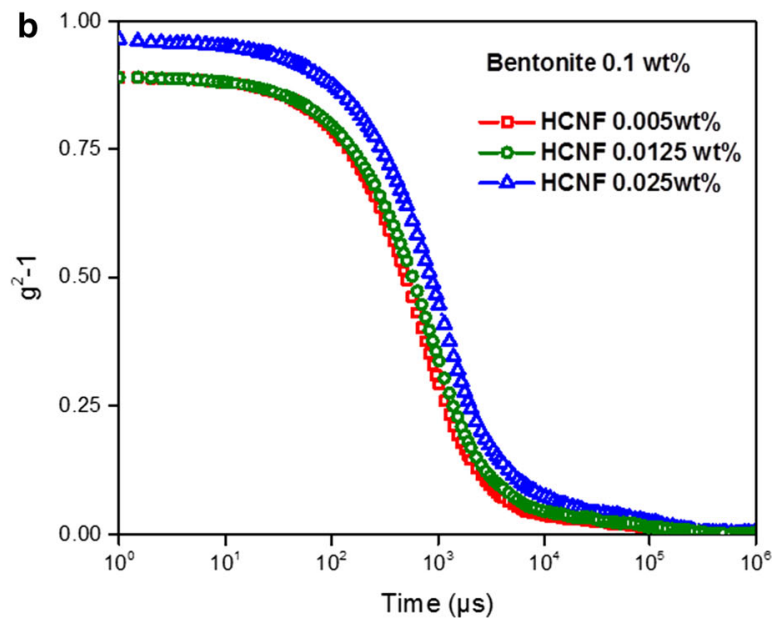

Fig. 7 Normalized autocorrelation function curves for diluted sepiolite (a) and bentonite (b) dispersions with varying HCNF concentrations 


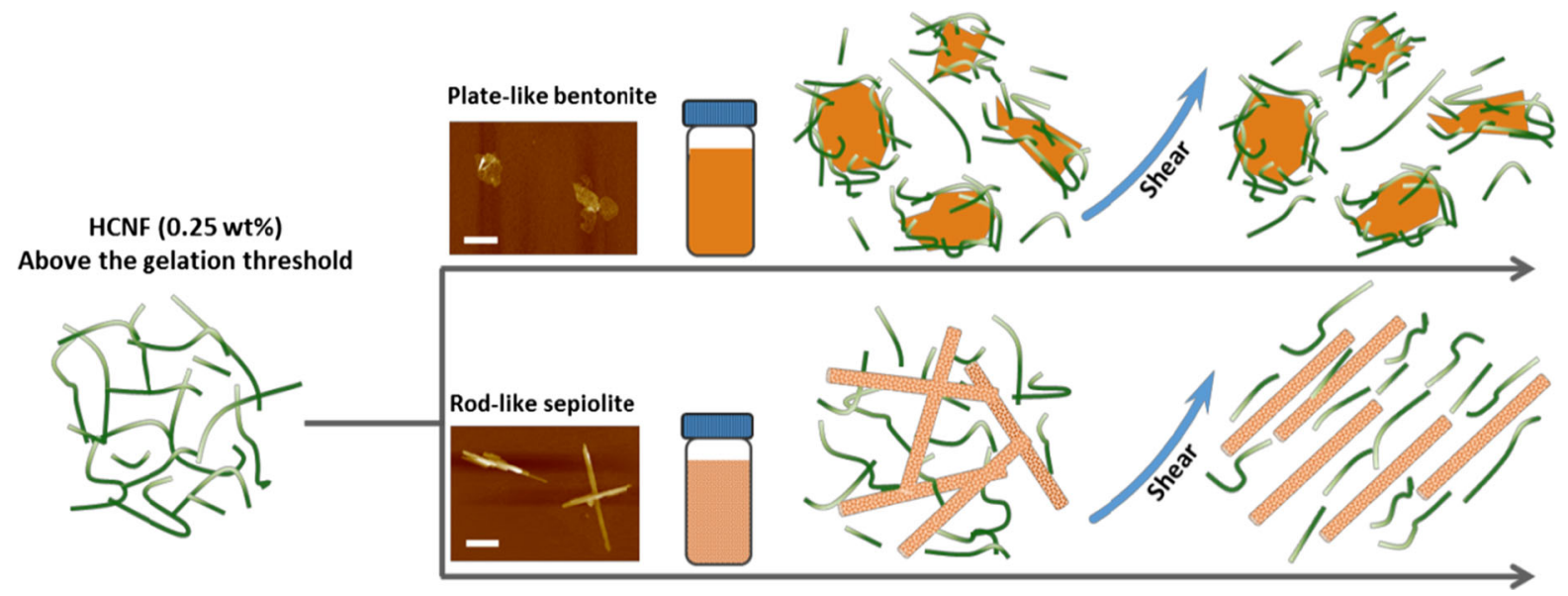

Fig. 8 Schematic of microstructure of $\mathrm{CNF}-$ nanoclay aqueous dispersions at quiescent conditions and under shear (scale bar in AFM images is $500 \mathrm{~nm}$ )

The viscosity ratio of the HCNF-sepiolite dispersion was more than 100 times higher on addition of $0.1 \mathrm{wt} \%$ HCNFs compared with addition of 0.25 or $0.5 \mathrm{wt} \%$ CNFs. In fact, the viscosity ratio of the HCNF-sepiolite $(0.1 \mathrm{wt} \%)$ dispersion was slightly higher than the relative viscosity of the sepiolite dispersion at shear rate of $10 \mathrm{~s}^{-1}$, which suggests that the sepiolite network in the dispersion was relatively unaffected by addition of this low content of HCNFs. The significant decrease of the viscosity ratio when adding more HCNFs ( 0.25 or $0.5 \mathrm{wt} \%)$ into the sepiolite dispersion indicates that addition of HCNFs at concentrations above the gelation threshold can promote breakup of the sepiolite network and assist in alignment of the rod-like clay particles under shear.

We also measured the time-intensity correlation function (TCF) of nanoclay dispersions with and without HCNFs. Figure 7 shows that the TCF curve for sepiolite was unaffected by addition of HCNFs, whereas the TCF curve for the bentonite dispersion shifted to longer decay times after addition of HCNFs. The TCF is related to the apparent hydrodynamic radius (particle diffusivity), which suggests that HCNFs adsorb onto the bentonite particles (Ferse et al. 2008). Indeed, previous work has shown that cellulose nanoparticles and cellulose-based polymers can be used to disperse clays in water (Ming et al. 2016; Sjöberg et al. 1999).

Figure 8 shows a schematic illustration of the interactions and shear-dependent microstructure of composite dispersions of $\mathrm{CNF}$ and bentonite or sepiolite.

\section{Conclusions}

Composite dispersions of CNFs and two different nanoclays, i.e., plate-like bentonite and rod-like sepiolite, displayed complex rheological behavior that depended on both the morphology of the clay particles and the interactions between the nanoclay and CNF particles at quiescent conditions and under shear. Dispersions of CNF displayed a gelation threshold and rheological properties that depended on the charge density. The lower gelation threshold, higher viscosity, and higher storage modulus of aqueous dispersions of CNFs with high compared with medium charge density were found to be related to the larger effective volume fraction and higher degree of defibrillation or dispersion. The more pronounced shear-thinning behavior of $2 \mathrm{wt} \%$ aqueous dispersions of sepiolite compared with bentonite suggests that the more weakly charged rod-like sepiolite particles form a stronger 3D network compared with the more highly charged, plate-like bentonite particles. Rheological studies on composite dispersions of CNFs and nanoclays suggested that the sepiolite network was relatively unaffected by addition of small amounts of CNFs, but addition of CNFs at concentrations above the gelation threshold promoted breakup of the sepiolite network and assisted in alignment of the rod-like clay particles also at low shear rate. The rheological properties of CNF-bentonite dispersions was less affected by addition of increasing amounts of CNFs compared with CNF-sepiolite dispersions. Dynamic light scattering (DLS) results showed that 
the CNFs adsorbs onto bentonite, resulting in an increase of the apparent hydrodynamic radius, while the DLS response of sepiolite was unaffected by addition of CNFs. We have shown that the complex rheological properties of CNF-nanoclay dispersions are influenced by the surface charge of the CNFs, the morphology of the clay, and the interactions between the CNFs and nanoclay.

Acknowledgments This work is supported by the Wallenberg Wood Science Center. The authors also thank Nathalie Lavoine and Michael Agthe, Stockholm University for valuable discussion and suggestions, as well as Maryam Ghanad from $\mathrm{KTH}$ for help with the microfluidizer.

Open Access This article is distributed under the terms of the Creative Commons Attribution 4.0 International License (http:// creativecommons.org/licenses/by/4.0/), which permits unrestricted use, distribution, and reproduction in any medium, provided you give appropriate credit to the original author(s) and the source, provide a link to the Creative Commons license, and indicate if changes were made.

\section{References}

Abdul Khalil HPS, Bhat AH, Ireana Yusra AF (2012) Green composites from sustainable cellulose nanofibrils: a review. Carbohydr Polym 87:963-979. doi:10.1016/j. carbpol.2011.08.078

Abend S, Lagaly G (2000) Sol-gel transitions of sodium montmorillonite dispersions. Appl Clay Sci 16:201-227. doi:10.1016/S0169-1317(99)00040-X

Agoda-Tandjawa G, Durand S, Berot S, Blassel C, Gaillard C, Garnier C, Doublier JL (2010) Rheological characterization of microfibrillated cellulose suspensions after freezing. Carbohydr Polym 80:677-686. doi:10.1016/j.carbpol. 2009.11.045

Bailey L, Lekkerkerker HN, Maitland GC (2015) Smectite clayinorganic nanoparticle mixed suspensions: phase behaviour and rheology. Soft Matter 11:222-236. doi:10.1039/ $\mathrm{c} 4 \mathrm{sm} 01717 \mathrm{j}$

Blakeney WR (1966) The viscosity of suspensions of straight, rigid rods. J Colloid Interface Sci 22:324-330. doi:10. 1016/0021-9797(66)90012-9

Cinar M, Can M, Sabah E, Karaguzel C, Celik M (2009) Rheological properties of sepiolite ground in acid and alkaline media. Appl Clay Sci 42:422-426. doi:10.1016/j.clay. 2008.04.010

de Kort DW, Veen SJ, Van As H, Bonn D, Velikov KP, van Duynhoven JPM (2016) Yielding and flow of cellulose microfibril dispersions in the presence of a charged polymer. Soft Matter 12:4739-4744. doi:10.1039/ C5SM02869H

Dimic-Misic K, Puisto A, Paltakari J, Alava M, Maloney T (2013) The influence of shear on the dewatering of high consistency nanofibrillated cellulose furnishes. Cellulose 20:1853-1864. doi:10.1007/s10570-013-9964-9

Dong H, Snyder JF, Williams KS, Andzelm JW (2013) Cationinduced hydrogels of cellulose nanofibrils with tunable moduli. Biomacromolecules 14:3338-3345. doi:10.1021/ bm400993f

Duan J, Gong S, Gao Y, Xie X, Jiang L, Cheng Q (2016) Bioinspired ternary artificial nacre nanocomposites based on reduced graphene oxide and nanofibrillar cellulose. ACS Appl Mater Interfaces 8:10545-10550. doi:10.1021/ acsami.6b02156

Fall AB, Lindstrom SB, Sundman O, Odberg L, Wagberg L (2011) Colloidal stability of aqueous nanofibrillated cellulose dispersions. Langmuir 27:11332-11338. doi:10. 1021/la201947x

Fall AB, Lindström SB, Sprakel J, Wågberg L (2013) A physical cross-linking process of cellulose nanofibril gels with shear-controlled fibril orientation. Soft Matter 9:1852-1863. doi:10.1039/c2sm27223g

Ferse B, Richter S, Eckert F, Kulkarni A, Papadakis CM, Arndt K-F (2008) Gelation mechanism of poly( $N$-isopropylacrylamide)—clay nanocomposite hydrogels synthesized by photopolymerization. Langmuir 24:12627-12635. doi:10.1021/la802162g

Gabr MH, Phong NT, Abdelkareem MA, Okubo K, Uzawa K, Kimpara I, Fujii T (2013) Mechanical, thermal, and moisture absorption properties of nano-clay reinforced nano-cellulose biocomposites. Cellulose 20:819-826. doi:10.1007/s10570-013-9876-8

Gordeyeva KS, Fall AB, Hall S, Wicklein B, Bergstrom L (2016) Stabilizing nanocellulose-nonionic surfactant composite foams by delayed Ca-induced gelation. J Colloid Interface Sci 472:44-51. doi:10.1016/j.jcis.2016.03. 031

Hakansson KM et al (2014) Hydrodynamic alignment and assembly of nanofibrils resulting in strong cellulose filaments. Nat Commun 5:4018. doi:10.1038/ncomms5018

Hamedi MM et al (2014) Highly conducting strong nanocomposites based on nanocellulose-assisted aqueous dispersions of single-wall carbon nanotubes. ACS Nano 8:2467-2476. doi:10.1021/nn4060368

Henriksson M, Berglund LA, Isaksson P, Lindström T, Nishino $\mathrm{T}$ (2008) Cellulose nanopaper structures of high toughness. Biomacromolecules 9:1579-1585. doi:10.1021/ bm800038n

Isogai A, Saito T, Fukuzumi H (2011) TEMPO-oxidized cellulose nanofibers. Nanoscale 3:71-85. doi:10.1039/ c0nr00583e

Iwamoto S, Isogai A, Iwata T (2011) Structure and mechanical properties of wet-spun fibers made from natural cellulose nanofibers. Biomacromolecules 12:831-836. doi:10.1021/ bm101510r

Johansson L-S, Tammelin T, Campbell JM, Setala H, Osterberg M (2011) Experimental evidence on medium driven cellulose surface adaptation demonstrated using nanofibrillated cellulose. Soft Matter 7:10917-10924. doi:10.1039/ C1SM06073B

Jowkarderis L, van de Ven TG (2015) Rheology of semi-dilute suspensions of carboxylated cellulose nanofibrils. Carbohydr Polym 123:416-423. doi:10.1016/j.carbpol.2015.01.067 
Klemm D, Kramer F, Moritz S, Lindstrom T, Ankerfors M, Gray D, Dorris A (2011) Nanocelluloses: a new family of naturebased materials. Angew Chem 50:5438-5466. doi:10. 1002/anie.201001273

Li MC, Wu Q, Song K, Qing Y, Wu Y (2015) Cellulose nanoparticles as modifiers for rheology and fluid loss in bentonite water-based fluids. ACS Appl Mater Interfaces 7:5006-5016. doi:10.1021/acsami.5b00498

Liu A, Walther A, Ikkala O, Belova L, Berglund LA (2011) Clay nanopaper with tough cellulose nanofiber matrix for fire retardancy and gas barrier functions. Biomacromolecules 12:633-641. doi:10.1021/bm101296z

Lourenço AF, Gamelas JAF, Nunes T, Amaral J, Mutjé P, Ferreira PJ (2016) Influence of TEMPO-oxidised cellulose nanofibrils on the properties of filler-containing papers. Cellulose. doi:10.1007/s10570-016-1121-9

Luckham PF, Rossi S (1999) The colloidal and rheological properties of bentonite suspensions. Adv Colloid Interface Sci 82:43-92. doi:10.1016/S0001-8686(99)00005-6

Martoia F, Dumont PJJ, Orgeas L, Belgacem MN, Putaux JL (2016) Micro-mechanics of electrostatically stabilized suspensions of cellulose nanofibrils under steady state shear flow. Soft Matter 12:1721-1735. doi:10.1039/ C5SM02310F

Mazeau K (2011) On the external morphology of native cellulose microfibrils. Carbohydr Polym 84:524-532. doi:10. 1016/j.carbpol.2010.12.016

Ming S, Chen G, Wu Z, Su L, He J, Kuang Y, Fang Z (2016) Effective dispersion of aqueous clay suspension using carboxylated nanofibrillated cellulose as dispersant. RSC Adv 6:37330-37336. doi:10.1039/C6RA03935A

Moon RJ, Martini A, Nairn J, Simonsen J, Youngblood J (2011) Cellulose nanomaterials review: structure, properties and nanocomposites. Chem Soc Rev 40:3941-3994. doi:10. 1039/c0cs00108b

Munier P, Gordeyeva K, Bergstrom L, Fall AB (2016) Directional freezing of nanocellulose dispersions aligns the rodlike particles and produces low-density and robust particle networks. Biomacromolecules 17:1875-1881. doi:10. 1021/acs.biomac.6b00304

Nechyporchuk O, Belgacem MN, Pignon F (2014) Rheological properties of micro-/nanofibrillated cellulose suspensions: wall-slip and shear banding phenomena. Carbohydr Polym 112:432-439. doi:10.1016/j.carbpol.2014.05.092

Nechyporchuk O, Belgacem MN, Pignon F (2015) Concentration effect of TEMPO-oxidized nanofibrillated cellulose aqueous suspensions on the flow instabilities and smallangle X-ray scattering structural characterization. Cellulose 22:2197-2210. doi:10.1007/s10570-015-0640-0

Pääkkö M et al (2007) Enzymatic hydrolysis combined with mechanical shearing and high-pressure homogenization for nanoscale cellulose fibrils and strong gels. Biomacromolecules 8:1934-1941. doi:10.1021/bm061215p

Quennouz N, Hashmi SM, Choi HS, Kim JW, Osuji CO (2016) Rheology of cellulose nanofibrils in the presence of surfactants. Soft Matter 12:157-164. doi:10.1039/ C5SM01803J

Ruiz-Hitzky E, Darder M, Fernandes FM, Wicklein B, Alcântara ACS, Aranda P (2013) Fibrous clays based bionanocomposites. Prog Polym Sci 38:1392-1414. doi:10. 1016/j.progpolymsci.2013.05.004

Sabah E, Mart U, Çınar M, Çelik MS (2007) Zeta potentials of sepiolite suspensions in concentrated monovalent electrolytes. Sep Sci Technol 42:2275-2288. doi:10.1080/ 15275920701313616

Saito T, Nishiyama Y, Putaux J-L, Vignon M, Isogai A (2006) Homogeneous suspensions of individualized microfibrils from TEMPO-catalyzed oxidation of native cellulose. Biomacromolecules 7:1687-1691. doi:10.1021/ bm060154s

Schütz C et al (2012) Hard and transparent films formed by nanocellulose- $\mathrm{TiO}_{2}$ nanoparticle hybrids. PLoS ONE 7:e45828. doi:10.1371/journal.pone.0045828

Siró I, Plackett D (2010) Microfibrillated cellulose and new nanocomposite materials: a review. Cellulose 17:459-494. doi:10.1007/s10570-010-9405-y

Sjöberg M, Bergström L, Larsson A, Sjöström E (1999) The effect of polymer and surfactant adsorption on the colloidal stability and rheology of kaolin dispersions. Colloids Surf A Physicochem Eng Asp 159:197-208. doi:10.1016/ S0927-7757(99)00174-0

Tanaka R, Saito T, Ishii D, Isogai A (2014) Determination of nanocellulose fibril length by shear viscosity measurement. Cellulose 21:1581-1589. doi:10.1007/s10570-014-0196-4

Tanaka R, Saito T, Hänninen T, Ono Y, Hakalahti M, Tammelin T, Isogai A (2016) Viscoelastic properties of core-shellstructured hemicellulose-rich nanofibrillated cellulose in dispersion and wet-film states. Biomacromolecules 17:2104-2111. doi:10.1021/acs.biomac.6b00316

Usov I et al (2015) Understanding nanocellulose chirality and structure-properties relationship at the single fibril level. Nat Commun 6:7564. doi:10.1038/ncomms8564

Wang J, Cheng Q, Lin L, Jiang L (2014) Synergistic toughening of bioinspired poly(vinyl alcohol)-clay-nanofibrillar cellulose artificial Nacre. ACS Nano 8:2739-2745. doi:10. 1021/nn406428n

Wicklein B, Kocjan A, Salazar-Alvarez G, Carosio F, Camino G, Antonietti M, Bergstrom L (2015) Thermally insulating and fire-retardant lightweight anisotropic foams based on nanocellulose and graphene oxide. Nat Nanotechnol 10:277-283. doi:10.1038/nnano.2014.248

Wicklein B, Kocjan D, Carosio F, Camino G, Bergström L (2016) Tuning the nanocellulose-borate interaction to achieve highly flame retardant hybrid materials. Chem Mater 28:1985-1989. doi:10.1021/acs.chemmater. 6b00564

Zheng Q, Cai Z, Gong S (2014) Green synthesis of polyvinyl alcohol (PVA) — cellulose nanofibril (CNF) hybrid aerogels and their use as superabsorbents. J Mater Chem A 2:3110. doi: $10.1039 / \mathrm{c} 3$ ta14642a 